\title{
Stability research on spinning of ultra-thin-wall aluminum alloy tube
}

\author{
Chengyong Wang ${ }^{1} \cdot$ Jin $\mathrm{Wu}^{1} \cdot$ Jinhong Chen ${ }^{1} \cdot$ Zhiwei Xiang $^{1} \cdot$ Xiaotao Sheng $^{1}$
}

Received: 14 November 2019 / Accepted: 15 April 2020 / Published online: 22 April 2020

(c) Springer Nature Switzerland AG 2020

\begin{abstract}
In this paper, the instability in spinning process of ultra-thin-wall tube is analyzed, and the influence law of different parameters of roller and main process parameters in spinning forming process. Firstly, the forward spinning finite element model of ultra-thin wall tube is established and its reliability is verified. It is concluded that the instability of ultra-thinwall aluminum alloy tube during spinning is caused by its poor rigidity and material flow disorder. The effects of different parameters of roller (roller diameters, roller forming angles and roller corner radii) and the main process parameters (feed rates, friction coefficients and thinning rates) on the equivalent plastic strain and diameter expansion amount in spinning forming process were investigated. The results show that the influence of each parameter on the stability of ultra-thin-wall is quite different. The influence of roller corner radius is stronger than roller diameter and much stronger than roller forming angle. The influence that feed rate does the expansion amount to exist a minimum value. The friction coefficient and thinning rate have positive correlation with expansion amount. The optimum combination of process parameters was further determined, and the accuracy of the results was verified by experiments, which provided reference and guidance for actual production.
\end{abstract}

Keywords Ultra-thin-wall · Spinning · Finite element method · Plastic forming

\section{Introduction}

Large-capacity ( $\mathrm{V}>2 \mathrm{~L}$ ) ultra-thin-wall aluminum alloy tube (wall thickness $0.3 \sim 1 \mathrm{~mm}$ ) is widely used in liquid or gas low-pressure packaging container. Because of poor rigidity, great thickness accuracy and structure property of the ultra-thin-wall tube, the difficulty of integral forming is greater [1]. As a kind of continuous local plastic forming technology, power spinning has become the preferred forming method for axisymmetric rotating parts due to its high flexibility, low energy consumption and high precision. Due to the poor rigidity of ultra-thin-wall tube and that spinning is a plastic forming process with nonlinear characteristics, it is necessary to study its forming stability in the process of spinning.

Many scholars have studied the causes of quality problems in spinning forming. C.C. Wong [2] studied the effect of roller path and geometry on metal flow in the spinning process of cylindrical parts. They concluded that the final shape of spinning process depends on roller geometry, feed rate and the amount of deformation. Shinde [3] found that the roll forming angle, feed rate and thinning rate are important process parameters affecting the diameter expanding and ovality of the steel tube in backward spinning. Xu and Zhang [4] obtained the distribution of stress and strain rate during spinning of tube parts by finite element method and concluded that the forming quality is determined by the size of tube and roller, feed, thickness reduction, the number of rollers and nose radius. Hua [5] analyzed the power spinning process of three rollers by mechanical model of 3D deformation, and studied the differences of the equivalent plastic strains in the defects of bell mouth, bulge, and diameter expansion. Fazeli [6] analyzed the influence of main process parameters on the

Jin Wu, wujin95@foxmail.com | 'School of Materials Science and Engineering, Hefei University of Technology, 193Tunxi Road, Hefei 230009, People's Republic of China. 
diameter expansion and wall thickness uniformity during spinning process, and gave an empirical model of the diameter expansion and wall thickness variation. Kemin and Zhen [7] analyzed the influence of process parameters on diametral growth by establishing 3D finite element model of tube spinning. The results show that the formation of bell mouth and the increase of diameter are mainly due to the increase of circumstantial stress and strain. With the decrease of thickness reduction, the diameter expansion amount of workpiece diameter decreases. Xue [8] found that the bell mouth and diameter expansion in spinning forming process of tube were mainly caused by abrupt changes in circumferential stress and strain. Zeng [9] analyzed the changing rule of the equivalent stress and equivalent plastic strains in spinning forming process of cylinder and found that the equivalent stress and the equivalent plastic strain increase with forming time. Furthermore, many scholars have studied the influence of process parameters on forming quality during spinning forming. M. Gur and J. Tirosh [10] proposed the theoretical algorithm of contact area of cylinder deformation zone, and analyzed the influence law of axial and circumferential contact length in radial contact zone on forming accuracy, and explored the influence of different process parameters on contact length. M. Joseph Davidson [11] analyzed the effects of various forward spinning process parameters (such as feed rate, depth of cut, starting, speed of the mandrel) on the quality of A6061 aluminum alloy tube, and optimum process parameters for good surface quality are proposed. Chen shi [12] put forward the comprehensive quality index of cylindrical parts in strong spinning, and studied the influence of key parameters on workpiece quality in process of cylindrical parts under power spinning with orthogonal design method, and obtained the optimal parameter combination.

Although there are many researches on spinning forming of tube parts, but all of them focused on the spinning process of tube with wall thickness more than $1 \mathrm{~mm}$. Due to its thin wall thickness, poor structural stiffness and less deformation constraints, the cold power spinning process of ultra-thin-wall tube is prone to various kinds of instability phenomena such as diameter enlargement and drum shape, it has not been investigated properly. And we attempt to study the forming stability in forward spinning process of ultra-thin-wall aluminum tube (wall thickness $<1 \mathrm{~mm}$ ) with large length-diameter ratio. Therefore, forward spinning finite element model of ultra-thin wall tube is established under the ABAQUS environment. And finite element model is verified from two angles of energy and velocity field. Through finite element model of ultra-thin-wall aluminum alloy tube in forward spinning forming, the causes of its instability are analyzed. And the effects of different parameters of roller and the main process parameters on spinning stability were investigated.

\section{Finite element modeling}

The power spinning of tube can be divided into forward spinning and backward spinning according to whether the flow direction of metal is consistent with axial movement of roller [13]. The paper will establish finite element model of forward spinning with a bottomed ultra-thinwall tube and study the influenced factors of spinning stability.

\subsection{Geometric model}

The assembly structure of mandrel and workpiece is shown in Fig. 1a. For ultra-thin-wall tube, tapered roller with small arc is specially designed, as shown in Fig. 1 b, c. A model is established by the pretreatment of ABAQUS, as shown in Fig. 2. The blank is set as three-dimensional entity, and divided into three-dimensional eight-node hexahedral reduced integral element (C3D8R). The number of grids is 13,280 . Simultaneously, adaptive grid technique is applied to reduce the severe distortion of grid in spinning simulation process [14].

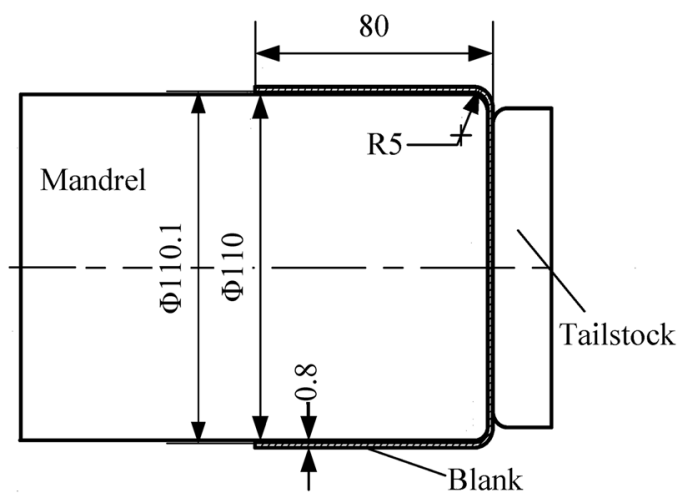

(a) Assembly drawing of mandrel and blank
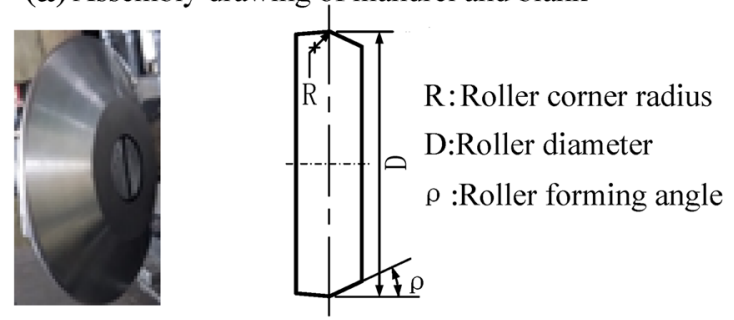

(b) Spinning roller

(c) Schematic diagram of spinning roller

Fig. 1 Mandrel, blank and spinning roller 


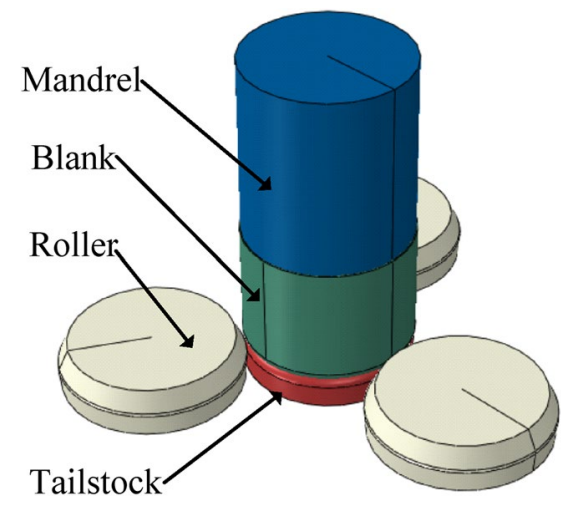

Fig. 2 Geometrical model of FEM

\subsection{Material properties}

Considering the pressure resistance of packaging container and the plastic requirement of spinning forming, aluminum alloy $\mathrm{A} 5052$ with good plasticity and medium strength is selected [15]. The stress-strain curve of A5052 is obtained through uniaxial tensile test, and the homogeneous plastic deformation is fitted with the curve of equation (2-1) [16].

$\sigma=K\left(\varepsilon_{0}+\varepsilon\right)^{n}-C$

Thus, material constitutive equation of aluminum alloy A5052 is shown in equation (2-2)

$\sigma=328.73(\varepsilon+0.0012)^{0.062}-28.2$

The specific material performance parameters are shown in Table 1.

\subsection{Boundary condition}

This paper adopts the mandrel rotation model that blank rotated under the clamping of mandrel and tailstock, and roller moved in the axial direction [17]. The specific loading method is that roller applies a constant axial velocity, and does not restrain the axial rotation degree. Therefore, roller rotates when subjected to the friction effect of blank. The friction types among mandrel, blank and roller all set to Coulomb friction [18].

Table 1 Material properties of A5052

\begin{tabular}{lllll}
\hline Material & $\begin{array}{l}\text { Density } \\
(\mathrm{kg} / \mathrm{m} \wedge 3)\end{array}$ & $\begin{array}{l}\text { Elasticity } \\
\text { modulus } \\
(\mathrm{GPa})\end{array}$ & Poisson ratio & $\begin{array}{l}\text { Yield strength } \\
(\mathrm{MPa})\end{array}$ \\
\hline A5052 & 2680 & 70 & 0.33 & 190 \\
\hline
\end{tabular}

\subsection{Model reliability verification}

In the finite element model of ABAQUS, verification is usually performed from two perspectives of energy and velocity field in order to ensure the reliability of the model. One hand, the ratio of kinetic energy of blank to total internal energy does not exceed $5 \% \sim 10 \%$ in forming process. The other hand, the velocity profile of deformed blank is smooth enough in spinning process $[19,20]$. The curves of kinetic energy and the ratio of kinetic energy to internal energy in finite element model are shown in Fig. 3. The figure shows the model has a reliable simulation result when the mass scaling factor is 500 .

\section{Preliminary analysis to causes of spinning instability}

The forward spinning of tube is essentially that tube achieves wall thickness and axial extension under spinning load [21]. Only when the metal produces uniform axial flow and flow to unformed area under spinning load, it can maintain the stability of spinning process. Otherwise, there will appear diameter expansion, accumulation, bulge, wrinkling and other spinning instabilities. Finally, these phenomena will affect the normal spinning process.

The poor rigidity of ultra-thin-wall tube and small circumferential and axial resistance when metal deformed at the contact point between roller and blank, which result in excessive metal flow to formed region or circumferential flow, thereby causing spinning instability. The final manifestation is expansion of formed zone. Tear will occur in the subsequent pass of spinning, which is a typical phenomenon of spinning instability, as shown in Fig. 4. In this paper, we try to quantitatively study the spinning stability

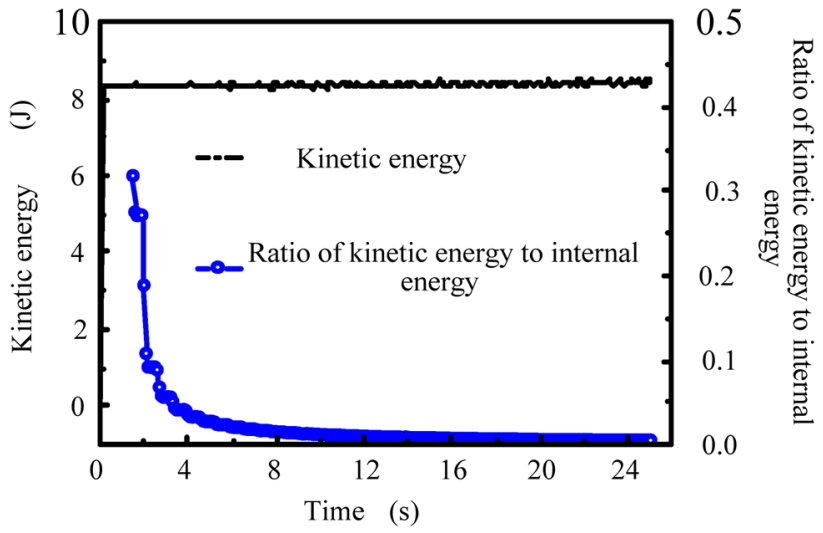

Fig. 3 Diagram of kinetic energy of blank and ratio of kinetic energy to internal energy of blank 


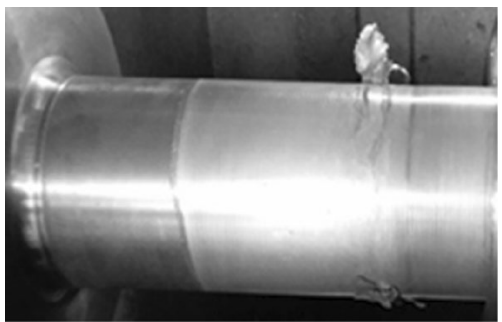

Fig. 4 Instability of film on spinning

of ultra-thin-wall aluminum alloy tube by equivalent plastic strain and diameter expansion.

\section{Parametric study on spinning instability of ultra-thin-wall tube}

There is a big difference between ultra-thin-wall tube and thick-wall tube on the spinning forming process. Therefore, the influences of roller geometry parameters and process parameters on spinning stability of ultra-thin-wall tube need further study. The initial parameters used in this paper are shown in Table 2.

\subsection{The influence of roller geometry parameters on spinning instability}

\subsubsection{Roller diameter}

This paper adopts the control variable method to study the influence of roller diameter on spinning instability. Figure 5 shows the distribution of the equivalent plastic strain at each moment on forward spinning. From the figure, there are three stages in forming process. The $A B$ segment is the starting stage. The roller is just in contact with the blank and the equivalent plastic strain increases sharply. The $B C$ segment is unsteady spinning stage. During the stage, spinning formation has just started and the stability is relatively poor. The $C D$ segment is stable spinning stage. The equivalent plastic strain remains basically unchanged in this stage. Meanwhile, as the roller diameter increases, the equivalent plastic strain also increases, indicating that the plastic deformation is more adequate.

Figure 6 shows the distribution of axial diameter expansion amount under the different roller diameters after

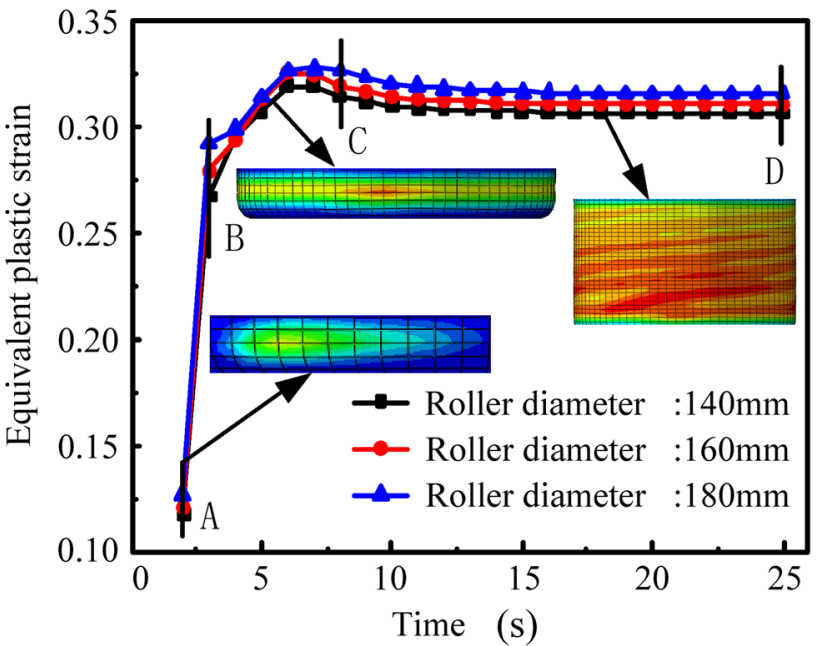

Fig. 5 Diagram of equivalent plasticity strain under different roller diameters with time

spinning process. It can be seen that as roller diameter increases, the diameter expansion amount decreases. The data of diameter expansion amount is further processed to obtain the maximum value, mean value and standard deviation of the expansion amount under the different roller diameters, as shown in Table 3. From the table, we can see that the diameter expansion is the largest when roller diameter is $140 \mathrm{~mm}$. The overall difference of diameter expansion between 160 and $180 \mathrm{~mm}$ is not obvious.

\subsubsection{Roller forming angle}

The main function of roller forming angle is to drive the material in front of roller to axial flow. Therefore, it has a certain influence on stability of forming. Figure 7 shows that distribution of equivalent plastic strain under difference roller forming angles at each period of spinning process. We can see from the figure, as roller forming angle increases, the equivalent plastic strain follow. The larger roller forming angle makes the gap of equivalent plastic strain in the axial direction also become larger, and spinning stability decrease. The possible reasons are that the increase of roller forming angle makes it easier to lead the material deform in front of roller. It is prone to stress concentration and bulging, resulting in local instable plastic flow at the contact area between roller and blank.

Table 2 Initial parameters for the model of spinning

\begin{tabular}{llllllll}
\hline Initial parameter & $\begin{array}{l}\text { Roller diameter } \\
(\mathrm{mm})\end{array}$ & $\begin{array}{l}\text { Roller forming } \\
\text { angle }\left(^{\circ}\right)\end{array}$ & $\begin{array}{l}\text { Roller corner } \\
\text { radius }(\mathrm{mm})\end{array}$ & Thinning ratio & $\begin{array}{l}\text { Spindle speed } \\
(\mathrm{r} / \mathrm{min})\end{array}$ & $\begin{array}{l}\text { Feed rate } \\
(\mathrm{mm} / \mathrm{r})\end{array}$ & $\begin{array}{l}\text { Friction } \\
\text { coefficient }\end{array}$ \\
\hline Magnitude & 160 & 20 & 1 & $25 \%$ & 120 & 1.6 & 0.2 \\
\hline
\end{tabular}




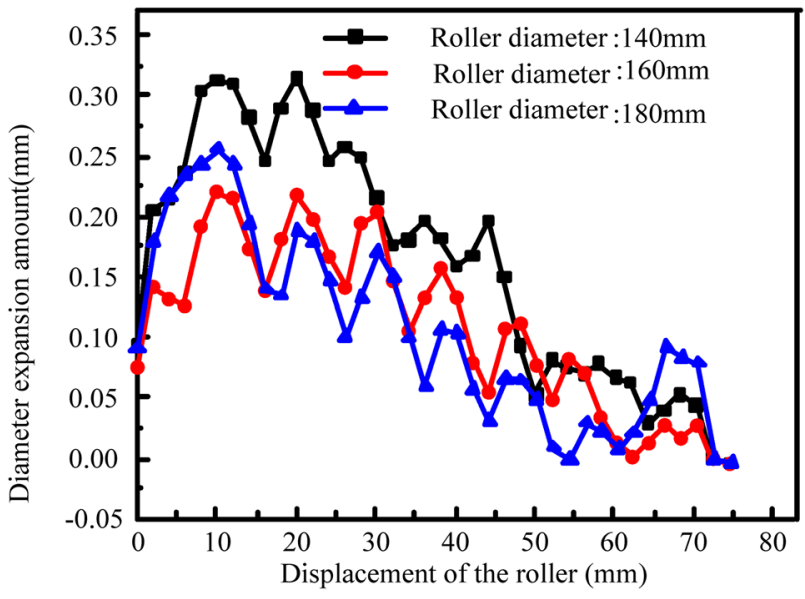

Fig. 6 Distribution of axial diameter expansion amount under different roller diameters

Table 3 Analysis table of diameter expansion amount under different roller diameters

\begin{tabular}{llll}
\hline $\begin{array}{l}\text { Roller diameter } \\
(\mathrm{mm})\end{array}$ & $\begin{array}{l}\text { Maximum value } \\
(\mathrm{mm})\end{array}$ & $\begin{array}{l}\text { Mean value } \\
(\mathrm{mm})\end{array}$ & $\begin{array}{l}\text { Standard } \\
\text { deviation } \\
(\mathrm{mm})\end{array}$ \\
\hline 140 & 0.313 & 0.163 & 0.0979 \\
160 & 0.22 & 0.108 & 0.0693 \\
180 & 0.256 & 0.106 & 0.0762 \\
\hline
\end{tabular}

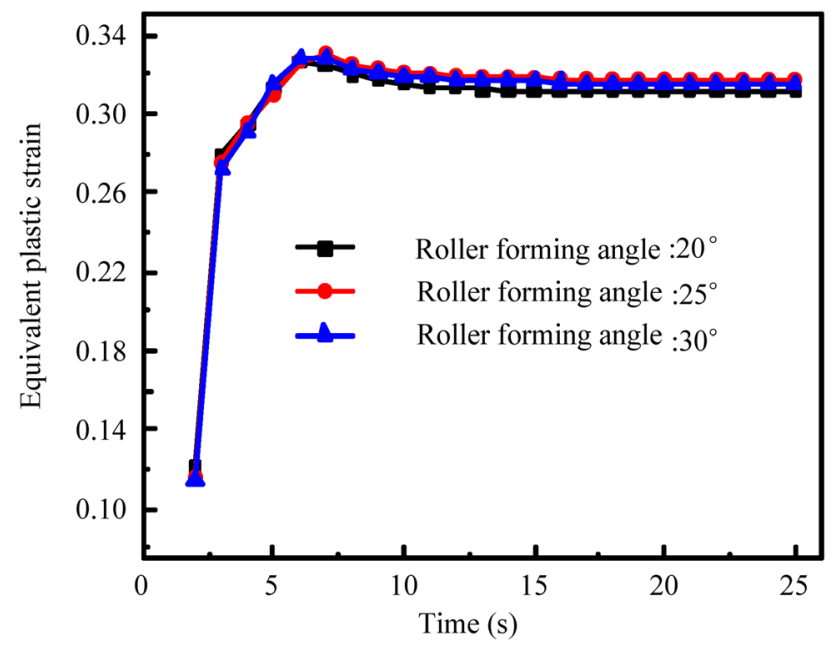

Fig. 7 Diagram of equivalent plasticity strain under different roller forming angles with time

Figure 8 shows the distribution of diameter expansion along the axial direction under different roller forming angles. As roller forming angle increases, the amount of diameter expansion increases to some extent. The amplitude of diameter expansion increases from $0.22 \mathrm{~mm}$ $\left(\rho=20^{\circ}\right)$ to $0.25 \mathrm{~mm}\left(\rho=30^{\circ}\right)$, and the amplification is

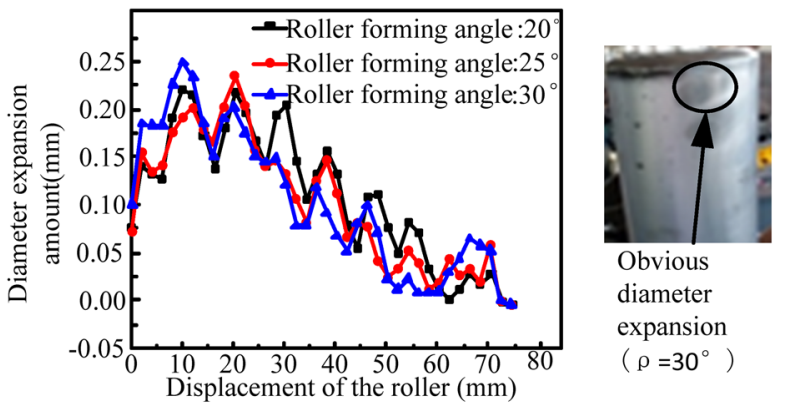

(a) The distribution of diameter expansion under (b) Expansion defect different roller forming angles

Fig. 8 The relation between diameter expansion and roller forming angles

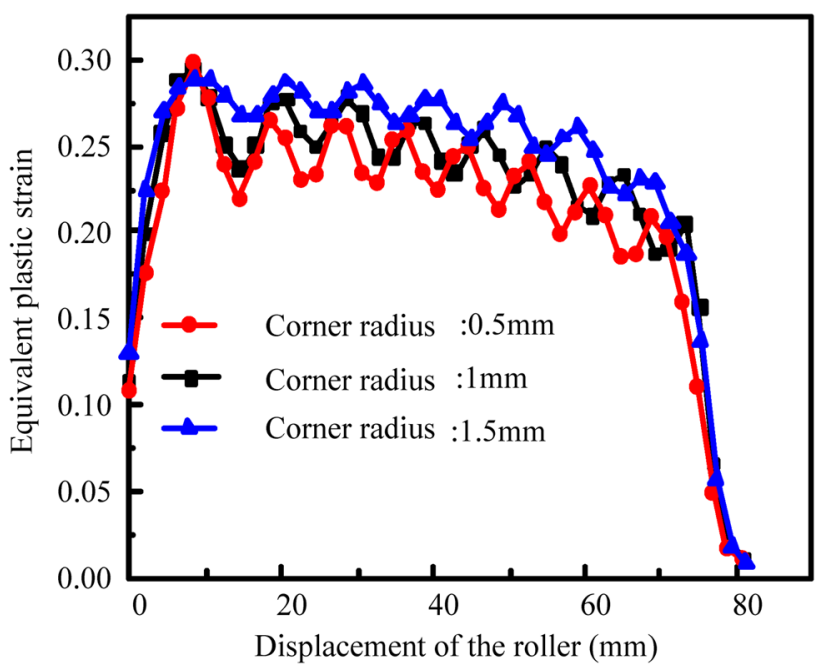

Fig. 9 Diagram of equivalent plasticity strain under different roller corner radii in axial direction

not obvious. When roller forming angle is $30^{\circ}$, the obvious expansion phenomenon will appear at the bottom of the cylinder wall, which lead to a poor forming quality. Therefore, when roller forming angle is $20^{\circ}$, the forming accuracy is greater.

\subsubsection{Roller corner radius}

The roller corner radius (R), as shown in Fig. $1 c$, will affect the size of contact area between roller and blank in spinning process. Then it will affect the stress-strain distribution in contact area, which is closely related to stability of spinning. The equivalent plastic strain along the axial direction is shown in Fig. 9. From the figure, it can be seen that equivalent plastic strain increases with roller corner radius, but the increase of maximum equivalent plastic strain is not obvious. From the results of finite element 
calculation, it can be seen the action area of maximum equivalent stress increases and a certain degree of stress concentration occurs. The possible reason is that with the increase of the roller corner radius, contact area between roller and blank also increase. The axial flow rate of material slows down, resulting in local material accumulation.

When the roller corner radius influences the stress and strain, it also will inevitably influence the dimension precision of ultra-thin-wall after spinning. The distribution of diameter expansion along certain axial direction under different roller corner radii is shown in Fig. 10. The figure shows that with the increase of roller corner radius, maximum diameter expansion also increases. The largest number of diameter expansion from $0.172 \mathrm{~mm}(\mathrm{R}=0.5 \mathrm{~mm})$ increases to $0.305 \mathrm{~mm}(\mathrm{R}=1.5 \mathrm{~mm})$, increases by nearly twice. It is bound to affect its subsequent forming stability.

\subsection{Influences of process parameters on spinning instability}

\subsection{1 (1) Feed rate.}

The feed rate refers to feed distance of the roller in the axial direction of tube when the mandrel rotates one revolution [22]. The change of feed rate will affect the distribution of stress and strain, and then affect the stability of spinning forming process. The equivalent plastic strain curve in the axial direction under different feed rates is shown in Fig. 11. It can be seen that with the increase of feed rate, the equivalent plastic strain decreases and the change is smoother. This is because the unformed region is in the free state during the forward spinning. The resistance in the front of the roller is less, the wall thickness is thinner and the rigidity is poorer. As the feed rate increases, the rate of material flows along the axial direction increase. As a result, its axial confinement effect is enhanced, and its overall deformation stiffness is further increased. Finally the plastic deformation becomes more uniform.

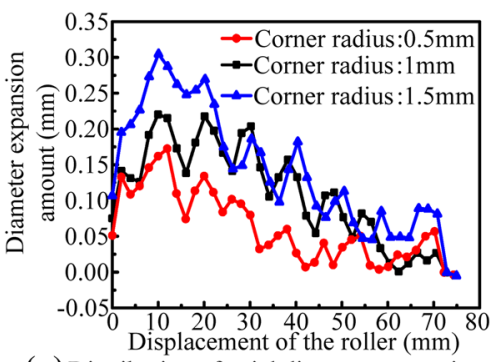

(a) Distribution of axial diameter expansion under different roller corner radii

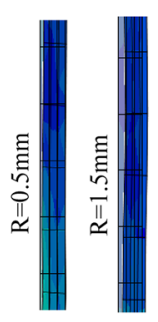

(b) Comparison of Diameter expansion conditions
Fig. 10 The relation between diameter expansion and roller corner radii

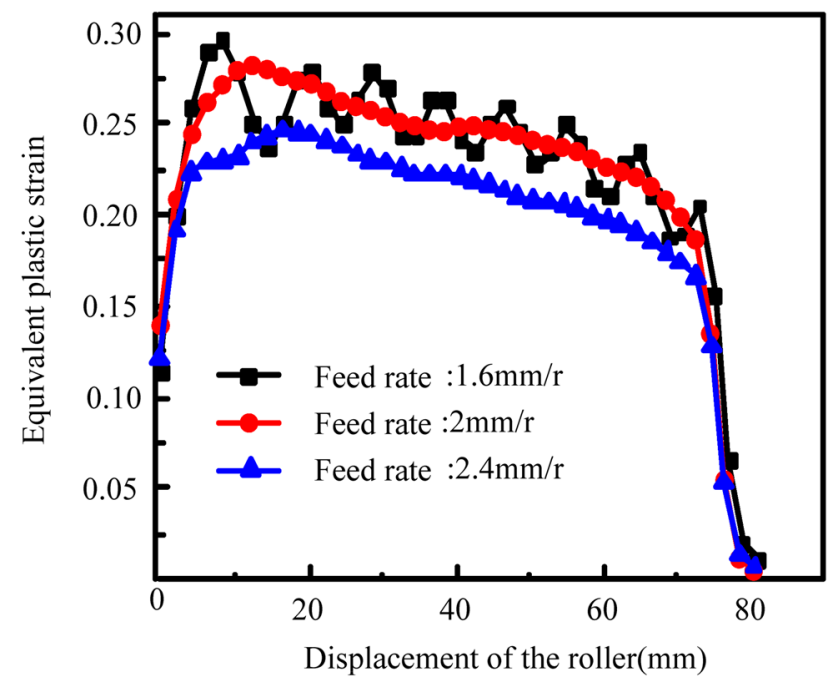

Fig. 11 Diagram of equivalent plasticity strain under different feed rates in axial direction

Figure 12 shows the distribution of the diameter expansion along the axial direction under different feed rates. It can be seen from the figure that the diameter expansion when the feed rate is $2.4 \mathrm{~mm} / \mathrm{r}$ is far greater than other feed rates. The diameter expansion is relatively small when the feed rate is $2 \mathrm{~mm} / \mathrm{r}$, which is suitable for power spinning forming of ultra-thin-wall. If the feed rate is too large or too small, the forming accuracy will be affected. Excessive feed rate causes the material in front of the roller to bugle, and then the flow of material will be disturbed. The excessively small feed rate makes the axial flow of the material smaller than its circumferential flow, so that the material flows too much to the bottom of the tube.

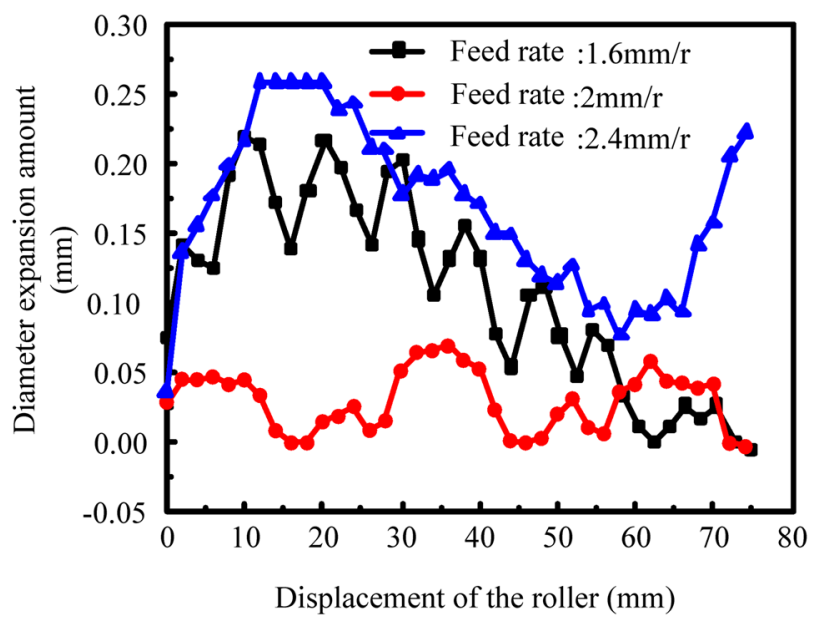

Fig. 12 Distribution of axial diameter expansion amount under different feed rates 
Finally the tube appears obvious diameter expansion phenomenon.

\subsection{2 (2) Lubrication condition.}

There are many kinds of complex contact states in the spinning process which in turn makes the friction state in the spinning forming process extremely complicated. The friction between the roller and blank is rolling friction, the friction coefficient is small and can be ignored. The friction between the mandrel and the blank is sliding friction, which has certain influence on the spinning stability. Figure 13 shows the diagram of equivalent plastic strain along the axial direction under different friction coefficients. From the figure, it can be seen that with the increase of the friction coefficient between the mandrel and the blank, the equivalent plastic strain decreases. This is because that as the internal friction coefficient increases, the frictional resistance between the mandrel and the blank increase. This in turn increases the flow resistance of material, resulting in the spinning stability reduce.

Figure 14 shows that the distribution of diameter expansion along the axial direction under different friction coefficients. It can be from the figure that the diameter expansion increases with the increase of friction coefficient at the bottom of the tube wall, and the amount of the diameter expansion in the mouth of tube wall is similar. Figure 15 is the analysis diagram of the diameter expansion under different friction coefficients. We can see from the figure that maximum value, mean value and standard deviation of the diameter expansion are not much different when the friction coefficient is 0.1 and 0.2. However, when the friction coefficient increases, the whole diameter expansion will increases. The phenomenon may be caused

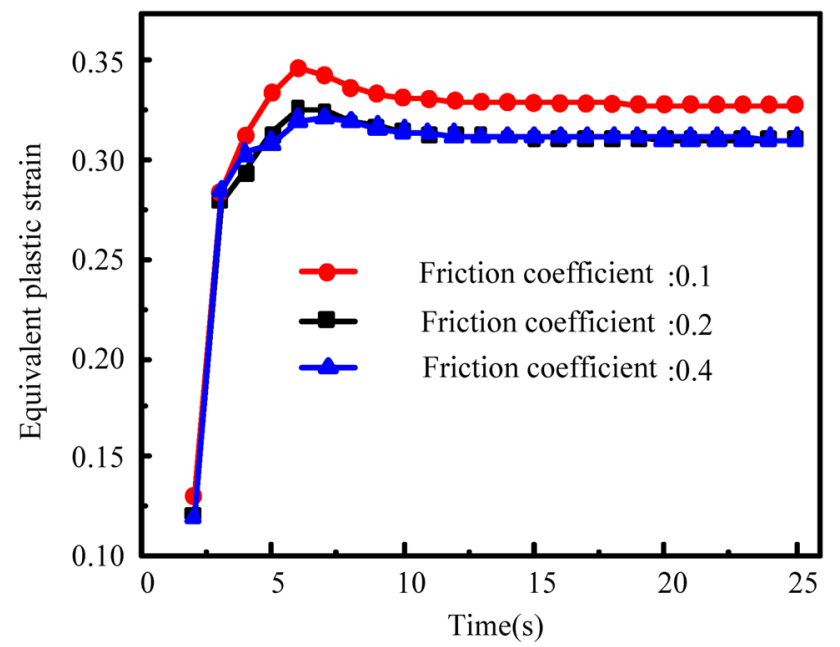

Fig. 13 Diagram of equivalent plasticity strain under different friction coefficients with time

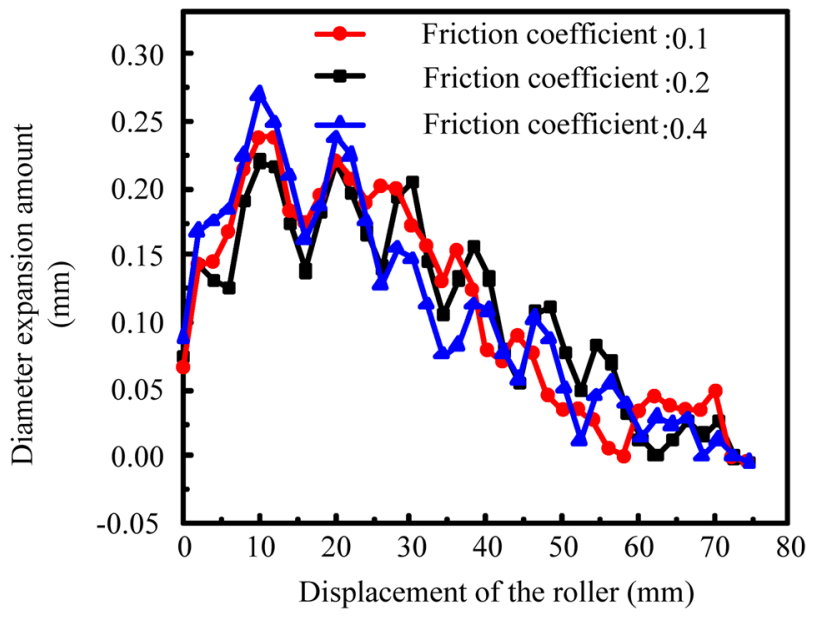

Fig. 14 Distribution of axial diameter expansion amount under different friction coefficient

by the chaotic axial flow of the material due to the excessive friction coefficient. The above analysis shows that the contact area between the roller and the blank should be properly lubricated to improve the flow of material.

\subsection{3 (3) Thinning ratio.}

The thinning ratio reflects the degree of spinning deformation, which is an important parameter influencing the diameter expansion in the spinning [23]. Figure 16 shows the distribution of equivalent plastic strain along the axial direction under the different thinning ratios. It can be seen from the figure that the equivalent plastic strain increases with the increase of the thinning ratio, and the gap of equivalent plastic strain increases. This is because that as the increase of the thinning ratio, the

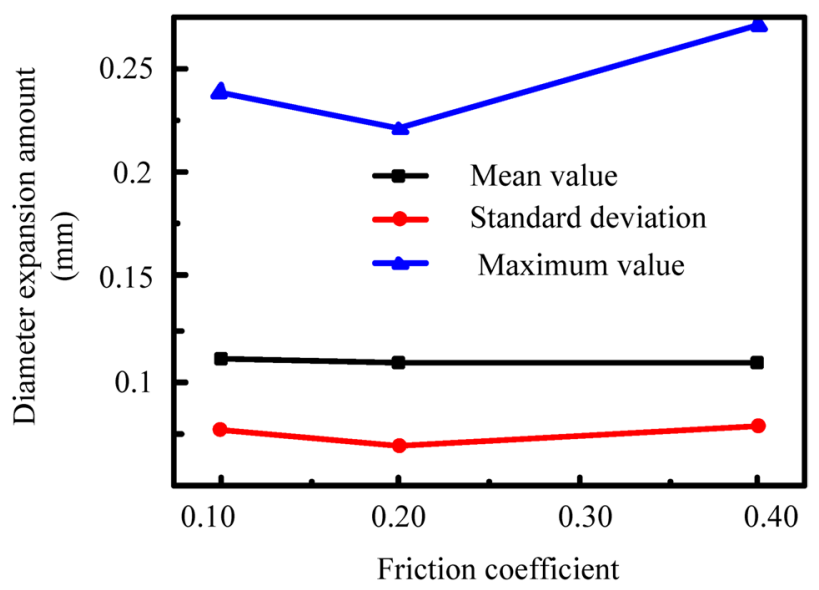

Fig. 15 Analysis diagram of diameter expansion amount under different friction coefficients 


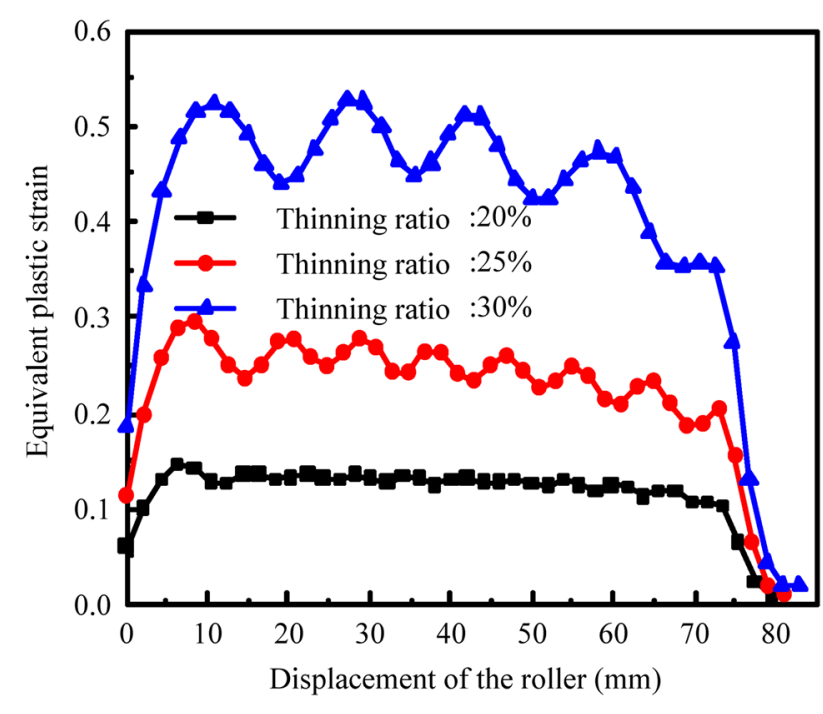

Fig. 16 Diagram of equivalent plasticity strain under different thinning ratios along axial direction

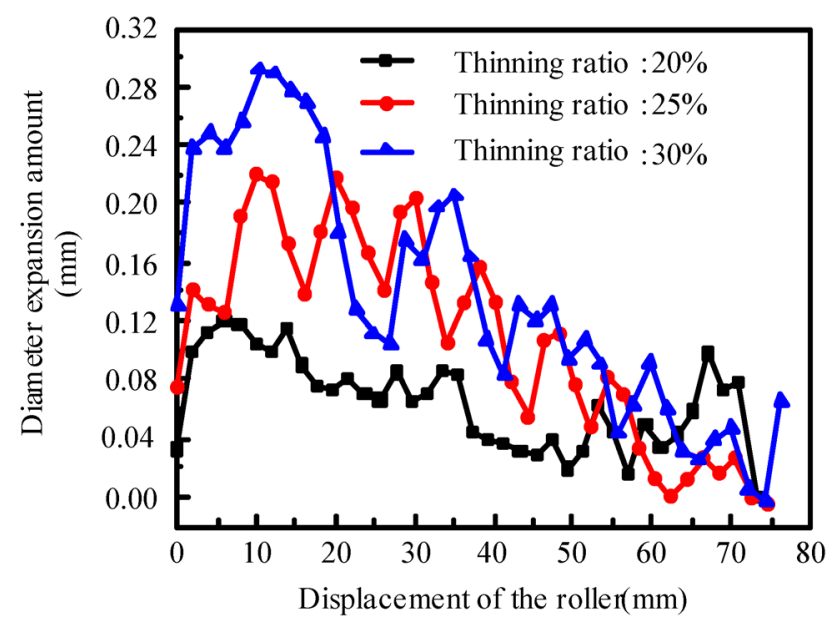

Fig. 17 Distribution of axial diameter expansion amount under different thinning ratios deformation degree is greater during the spinning process. This leads to more violent deformation of material and even spinning instability.

Figure 17 shows the distribution of diameter expansion under the different thinning ratios. We can see from the figure that as the thinning ratio increases, the diameter expansion also increases. The maximum diameter expansion increases from 0.12 to $0.29 \mathrm{~mm}$. When the thinning ratio is $20 \%$, the overall trend of the diameter expansion is relatively stable. With the increase of the thinning ratio, the axial distribution deviation of the diameter expansion has a larger deviation value, which is more prone to spinning instability.

\section{Experimental verification}

The forward spinning test of the ultra-thin-wall aluminum alloy tube was performed on the optimized combination of the simulations. Through the macroscopic observation of the forming quality of the inner and outer sides of the formed ultra-thin-wall tube, it was found that the diameter expansion is very good under this set of process combinations, but there are obvious cutting marks on the outside of the tube wall, as shown in the Fig. 18a. This is due to the fact that the roller corner radius is too small under the set of process parameters, resulting in a more similar cutting state during the forming process. In order to ensure the better surface quality while ensuring fittability, it is necessary to increase the roller corner radius. Therefore, the corner radius was increased to $1 \mathrm{~mm}$ and the spinning experiment performed again. We find that the surface quality and fittability are both good in the set of process parameters which is shown in the Fig. 18b.
Fig. 18 The charts of experimental results

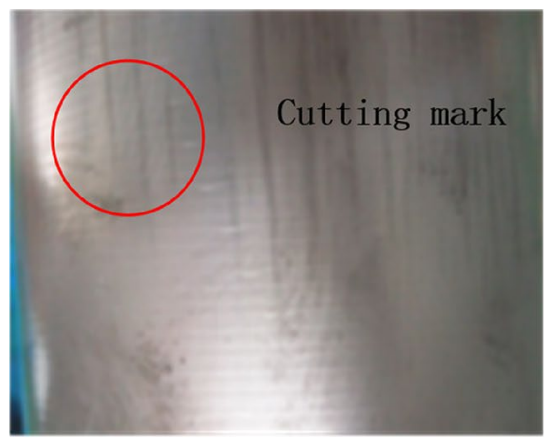

(a) $\mathrm{R}=0.5 \mathrm{~mm}$

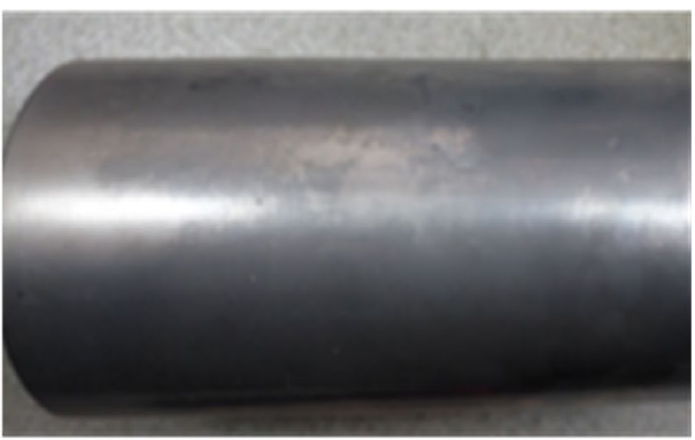

(b) $\mathrm{R}=1 \mathrm{~mm}$ 


\section{Conclusions}

The influence of roller geometric parameters of roller on the spinning stability was studied. It is found that with the increase of the roller diameter, the equivalent plastic strain increases and the amount of diameter expansion decreases. With the increase of the roller forming angle, the equivalent plastic strain increases but is not obvious. The amount of the diameter expansion does not change significantly. With the increase of the roller corner radius, the equivalent plastic strain increases and the diameter expansion increases obviously. Through these analyses, we found that the influence of the roller corner radius is greater than that of the roller diameter, and it is much larger than that of the roller forming angle. The influence of spinning process parameters on the spinning stability was studied. As the feed rate increases, the equivalent plastic strain decreases. Too large or too small feed rate will lead to the excessive diameter expansion. With the increase of the friction coefficient, the equivalent plastic strain decreases and the diameter expansion increases. As the thinning ratio increases, the equivalent plastic strain increases, and the diameter expansion also increases.

Through comparative analysis on multiple sets of parameters, the optimal combination of process parameters of spinning stability is obtained. The roller diameter is $160 \mathrm{~mm}$, the roller forming angle is $20^{\circ}$, the roller corner radius is $0.5 \mathrm{~mm}$, the feed rate is $2 \mathrm{~mm} / \mathrm{r}$, the friction coefficient is 0.2 , the thinning ratio is $20 \%$. Through experiment performs on the set of process parameters obtained by simulation. It was found that the fittability is better under this set of process parameters, but the cutting marks may appear. Therefore, the roller corner radius is further improved to improve the surface quality while ensuring fittability.

However, this paper only studied the effects of a few common parameters on spinning stability and the influence of temperature is ignored in the finite element simulation. In the future, the influence of changes in microstructure and mechanical properties on the pressure resistance of ultra-thin-wall tube after spinning forming process can be explored.

Acknowledgements This research was financially supported by the National Natural Science Foundation of China (Grant Nos.: 51275146). The authors are also grateful for the experimental platform of Suzhou Haoyuanda Technology Co., Ltd.

\section{Compliance with ethical standards}

Conflict of interest On behalf of all authors, the corresponding author states that there is no conflict of interest.

\section{References}

1. Xinhe Li, Yanping L, Yuesheng Y et al (2012) Analysis on bulging during flow forming of tube with ultra-thin-wall and large diameter-thickness ratio. J Plast Eng 19(3):64-70

2. Wong CC, Lin J, Dean TA (2005) Effects of roller path and geometry on the flow forming of solid cylindrical components. J Mater Process Technol 167:344-353

3. Shinde H, Mahajan P, Singh AK et al (2016) Process modeling and optimization of the staggered backward flow forming process of maraging steel via finite element simulations. Int J Adv Manuf Technol 87(5-8):1-14

4. Xu ZS (2001) 3D rigid-plastic FEM numerical simulation on tube spinning. J Mater Process Technol 113:710-713

5. Hua FA, Yang YS, Zhang YN, Guo MH, Guo DY, Tong WH, Hu ZQ (2005) Three-dimensional finite element analysis of tube spinning. J Mater Process Technol 168(1):68-74

6. Fazeli AR, Ghoreishi M (2011) Statistical analysis of dimensional changes in thermomechanical tube-spinning process. Int J Adv Manuf Technol 52(5-8):597-607

7. Kemin X, Zhen W, Yan L, Kezhi L (1997) Elasto-plastic FEM analysis and experimental study of diametral growth in tube spinning. J Mater Process Technol 69:172-175

8. Xue K, Zhen W, Yan L et al (1997) Elasto-plastic FEM analysis and experimental study of diametral growth in tube spinning. J Mater Process Technol 69(1-3):172-175

9. Zeng R, Ma F, Huang L, Li JJ (2015) Investigation on spinnability of profiled power spinning of aluminum alloy. Int J Adv Manuf Technol 80:535-548

10. Gur M, Tirosh J (1982) Plastic flow instability under compressive loading during shear spinning. J Eng Ind 104(1):17-22

11. Balasubramanian $K$, Tagore $G$ (2008) An experimental study on the quality of flow-formed AA6061 tubes. J Mater Process Technol 203(1):321-325

12. Chen Shi (2015) The analysis and optimization of key parameters in tube spinning process. Dissertation, Zhejiang University

13. Zhang $T$ (2009) Spinning forming process. Chemical Industry Press, Beijing

14. Mei Z, Feng S, Qiang D et al (2014) Forming mechanism and rules of mandreless neck-spinning on corrugated pipes. J Plast Eng 21(2):108-115

15. Hui C (2011) Research progress of 5052 aluminum alloy materials. Alum Fabr 6:33-39

16. Miao $P, Z$ Zhu $X$, Hu C et al (2014) Finite element modeling and optimization analysis on power spinning of the thin-wall cylinder. Forg Stamp Technol 39(9):137-142

17. Zhan M, Guo J, Fu MW et al (2017) Formation mechanism and control of flaring in forward tube spinning. Int J Adv Manuf Technol 94(14):1-14

18. Zhiren $H$, Hua $T$, Yun $H$ (2005) Study on roller rotation in element analysis of spinning forming. J Mech Strength 27(5):699-702 
19. Min Shi (2015) Study on baffle-assistant new spinning process for thin-walled aluminum alloy vessel head. Dissertation, Shanghai Jiao Tong University

20. Mohebbi MS, Akbarzadeh A (2010) Experimental study and FEM analysis of redundant strains in flow forming of tubes. J Mater Process Technol 210(2):389-395

21. Shuyong J, Zhengyi R (2008) Analysis of mechanics in ball spinning of thin-walled tube. Chin J Mech Eng 21(1):25-30

22. Lipeng $Z$, Zhichong $L$ (2006) Analysis of power spinning process parameters selection mode for cylinders. Forg Stamp Technol 31(1):43-45
23. Yujing Z, Xiuquan C, Qinxiang X (2011) Research on stepped spinning process of long and slim thin-wall tube. Light Alloy Fabr Technol 39(8):30-34

Publisher's Note Springer Nature remains neutral with regard to jurisdictional claims in published maps and institutional affiliations. 